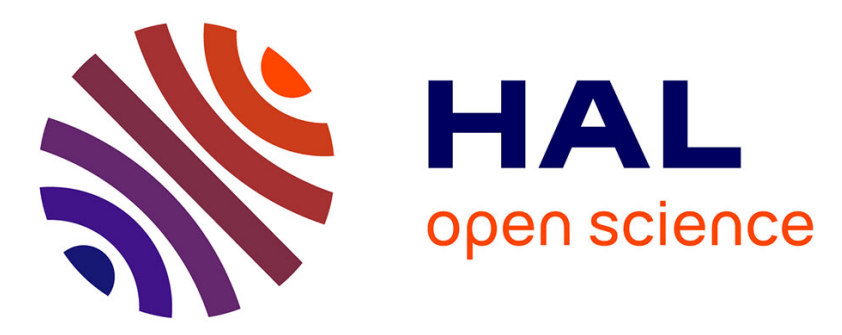

\title{
Eidolon: Visualization and Computational Framework for Multi-Modal Biomedical Data Analysis
}

Eric Kerfoot, Lauren Fovargue, Simone Rivolo, Wenzhe Shi, Daniel Rueckert, David Nordsletten, Jack Lee, Radomir Chabiniok, Reza Razavi

\section{To cite this version:}

Eric Kerfoot, Lauren Fovargue, Simone Rivolo, Wenzhe Shi, Daniel Rueckert, et al.. Eidolon: Visualization and Computational Framework for Multi-Modal Biomedical Data Analysis. 7th International Conference on Medical Imaging and Augmented Reality (MIAR 2016), Aug 2016, Berne, Switzerland. hal-01346357

\section{HAL Id: hal-01346357 https://hal.science/hal-01346357}

Submitted on 18 Jul 2016

HAL is a multi-disciplinary open access archive for the deposit and dissemination of scientific research documents, whether they are published or not. The documents may come from teaching and research institutions in France or abroad, or from public or private research centers.
L'archive ouverte pluridisciplinaire HAL, est destinée au dépôt et à la diffusion de documents scientifiques de niveau recherche, publiés ou non, émanant des établissements d'enseignement et de recherche français ou étrangers, des laboratoires publics ou privés. 


\title{
Eidolon: Visualization and Computational Framework for Multi-Modal Biomedical Data Analysis
}

\author{
Eric Kerfoot ${ }^{1}$, Lauren Fovarge ${ }^{1}$, Simone Rivolo ${ }^{1}$, Wenzhe $\mathrm{Shi}^{2}$, Daniel \\ Rueckert $^{2}$, David Nordsletten ${ }^{1}$, Jack Lee ${ }^{1}$, Radomir Chabiniok ${ }^{1,3}$, and Reza \\ Razavi $^{1}$ \\ 1 Division of Imaging Sciences and Biomedical Engineering, \\ King's College London, London, UK \\ 2 Department of Computing, Imperial College London, London, UK \\ ${ }^{3}$ Inria and Paris-Saclay University, Palaiseau, France
}

\begin{abstract}
Biomedical research, combining multi-modal image and geometry data, presents unique challenges for data visualization, processing, and quantitative analysis. Medical imaging provides rich information, from anatomical to deformation, but extracting this to a coherent picture across image modalities with preserved quality is not trivial. Addressing these challenges and integrating visualization with image and quantitative analysis results in Eidolon, a platform which can adapt to rapidly changing research workflows. In this paper we outline Eidolon, a software environment aimed at addressing these challenges, and discuss the novel integration of visualization and analysis components. These capabilities are demonstrated through the example of cardiac strain analysis, showing the Eidolon supports and enhances the workflow.
\end{abstract}

\section{Introduction}

A growing area of current biomedical research involves the computational analysis of multi-modal imaging data (MRI, CT, etc.) to extract a greater understanding of biological behaviour and the nature of pathologies. For example, in cardiovascular research, different imaging sequences of a patient provide pieces of an overall picture, such as geometry, 3D motion, or scar and fibrosis. Although there have been considerable advances in correcting motion artefacts in acquisition, the current state of the art still requires substantial post-processing for the detailed quantification needed in biomedical engineering. Done correctly, this can result in the characterization of patient-specific properties or the derivation of innovative biomarkers, giving a deeper understanding of disease than can be achieved by visual assessment of the images.

Visualization is a key component in this process, used to verify the correctness of image reconstruction, image fusion, quantification, and to display the results of simulations or other derived calculations. This requires a visualization environment which can seamlessly render multiple time-dependent image 

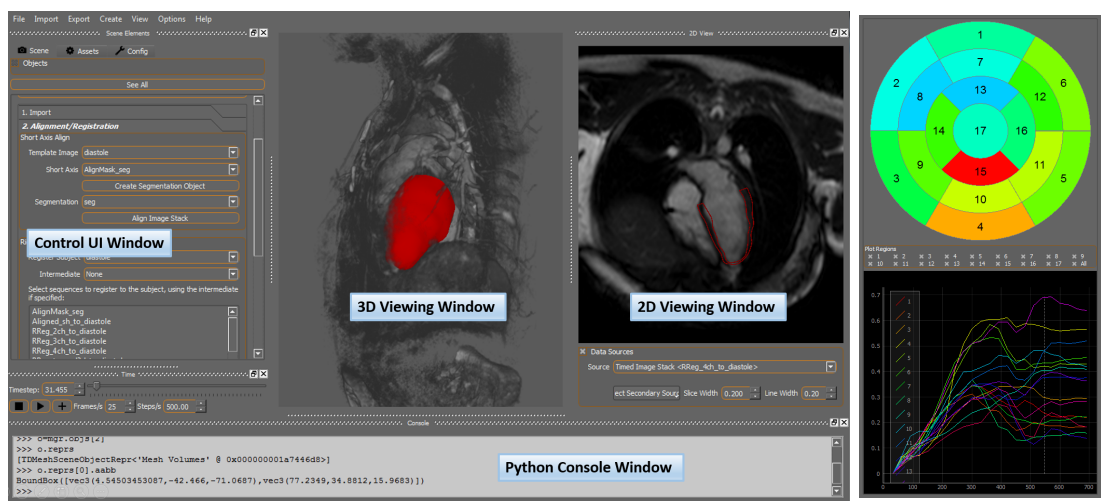

Fig. 1. Screenshot of the Eidolon's user interface. Left image illustrates the control interface, 3D view, 2D view, and Python console. Here three different representations of the left ventricle (LV) are shown, two image sequences and one geometric mesh. Right image illustrates the analysis pane, an alternative to the $2 \mathrm{D}$ viewing window, showing calculated strains in AHA regions of the LV.

or geometry objects simultaneously in a single scene. Data processing and computation is a second component in the process which is necessary to manipulate datasets, automate common tasks in a workflow, and otherwise prototype the research activities. Thus, exposing data processing functionality through an interactive programming interface is a necessary tool for these activities. As a result there exists a compelling need for an integrated environment where visualization, data processing, and prototyping are presented through a common graphical and programming interface.

In this paper we present our software, Eidolon, which addresses these requirements with a unique integrated visualization and computing application, shown in Figure 1. The important advantage for our software is the integration of an image processing and quantitative analysis environment with visualization components specifically tailored to bioengineering applications of medical image data. This allows users to rapidly process and experiment with data in a way more general tools cannot support. Moreover, it bridges the gap between biomedical engineers and clinicians by providing a common platform, used by the former for developing workflows and by the latter for multi-modal imaging patient evaluations.

This visualization component is implemented in $\mathrm{C}++$ and based on the Ogre3D engine(http://www.ogre3d.org). The majority of Eidolon however is implemented in the Python language (http://www.python.org) using the SciPy library stack (http://www.scipy.org) to include a significant amount of the processing and computational facilities. Eidolon is thus built upon large open-source projects and is itself open source.

Initially the paper will describe how Eidolon is tailored to biomedical research and the features designed to facilitate research activities. Next, the utilization of Eidolon will be illustrated through a specific research workflow (strain 


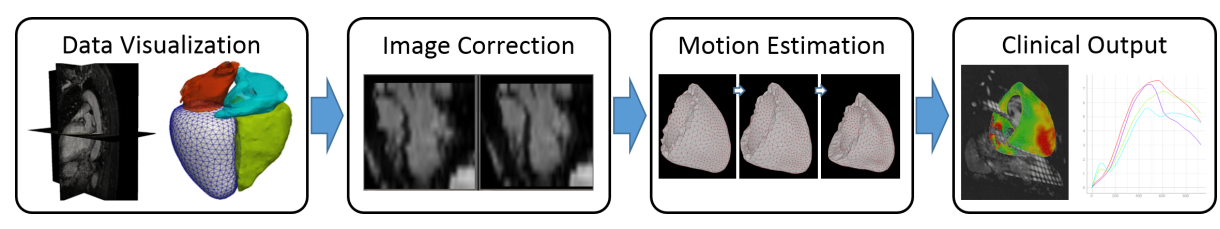

Fig. 2. Illustration of overall workflow for biomedical engineers assessing image motion derived outputs.

evaluation for a patient with dyssynchronous heart failure), followed by a concluding discussion and future work.

\section{Method}

For biomedical engineers focused on using multi-modal imaging for the derivation of clinically relevant indicators, the overall workflow can be broken into four main stages, illustrated in Figure 2. Image data is firstly acquired and visualized, correction operations are then applied to the data to account for acquisition motion error. Subsequently, parameters such as regional strain and torsion are estimated from time-dependent images, and clinical assessment is made.

In this section we outline how Eidolon is specifically tailored to this workflow by providing integrated imaging processing functionality, i.e. image registration and alignment with motion tracking, and comprehensive visualization features. Eidolon implements much of its image reconstruction features with the Image Registration Toolkit (IRTK) [812, creating an accessible GUI designed for a non-technical clinician audience.

Other visualization and biomedical platforms exist to address similar requirements (compared in Table 1), however for the purposes of medical imaging and biomedical engineering Eidolon provides the most comprehensive functionality in a single environment. Combining these features with an user-friendly interface results in a tool which maximizes the efficiency of the biomedical workflow illustrated above.

Unlike the other tools compared in Table 1, Eidolon is primarily implemented in the Python language with $\mathrm{C}++$ reserved for the critical rendering facilities only. This allows Eidolon to take advantage of the dynamic and malleable software environment Python provides through the nature of the language itself, and integrate with the very large standard library the Python runtime includes and numerous other third party packages. This capability will ensure Eidolon users are using the most state-of-the-art algorithms in image correction.

\subsection{Image Correction}

A significant component of data processing involves the spatio-temporal correction of image data through alignment and registration. IRTK is an open-source 
Table 1. Comparison of Major Biomedical Software Libraries and Applications

\begin{tabular}{|c|c|c|c|c|c|c|c|c|c|c|}
\hline & 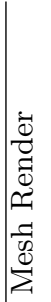 & 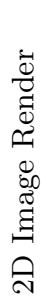 & 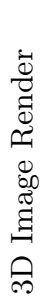 & 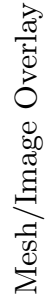 & 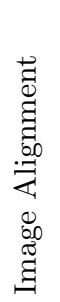 & 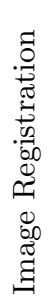 & 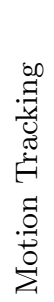 & 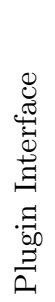 & 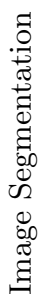 & 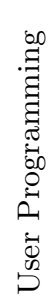 \\
\hline ParaView & $x$ & $x$ & $x$ & $x$ & . & . & . & $x$ & . & $x$ \\
\hline Osirix & . & $x$ & $x$ & . & . & . & . & $x$ & . & . \\
\hline medINRIA & $x$ & $x$ & $x$ & $x$ & . & $x$ & . & $x$ & $x$ & . \\
\hline 3D Slicer &. & $x$ & $x$ & . & . & . & . & $x$ & $x$ & . \\
\hline MITK & $x$ & $x$ & $x$ & $x$ & . & . & . & $x$ & $x$ & . \\
\hline MeVisLab & $x$ & $x$ & $x$ & $x$ & . & $x$ & . & $x$ & $x$ & $x$ \\
\hline cmgui & $x$ & $x$ & . & . & . & . & . & . & . & $x$ \\
\hline ITK-Snap & $x$ & $x$ & . & . & . & . & . & . & $x$ & . \\
\hline GIMIAS & $x$ & $x$ & $x$ & $x$ & . & . & . & $x$ & . & . \\
\hline Seg3D & . & $x$ & $x$ & . & . & . & . & $x$ & $x$ & . \\
\hline Eidolon & $x$ & $x$ & $x$ & $x$ & $x$ & $x$ & $x$ & $x$ & $x$ & $x$ \\
\hline
\end{tabular}

library of algorithms which achieves the main post-processing tasks : rigidly registering one image series to another, and correcting alignment of the individual slices of a multi-slice sequence (eg. short axis Cine stack). This ability to align data is especially critical for quantitative analyses, where continuous and smooth spatial descriptions are needed. Eidolon provides a graphical user interface for these algorithms, allows researchers and clinicians to easily utilize this functionality, where alternative GUI options are limited.

Figure 3 illustrates an example of rigid registration between two different image sequences. Image $3 \mathrm{a}$ shows a long axis $2 \mathrm{D}$ image on the left and $3 \mathrm{D}$ volume on the right, both representing the left ventricle of the same subject. Although the two images are acquired as different series, they are part of one study and so should correlate in space. However they are clearly misaligned, a common issue due to breathold variation or patient movement. To compensate, IRTK's rigid registration is applied to correctly re-align the images, giving more continuous data as shown in image $3 \mathrm{~b}$.

Figure 3r shows the alignment of different slices on a single image series, the cardiac Cine MRI short axis. This is a sequence of 5-10 mm thick images which spatially and temporally cover the heart's ventricles. The acquisition of the series requires multiple breatholds by the patient and thus variability in chest position causes misalignment, as seen here in the jagged appearance of the blood pool. Post-processing through Eidolon can easily correct this misalignment with the wrapped IRTK functionality as shown in image $3 \mathrm{~d}$. 

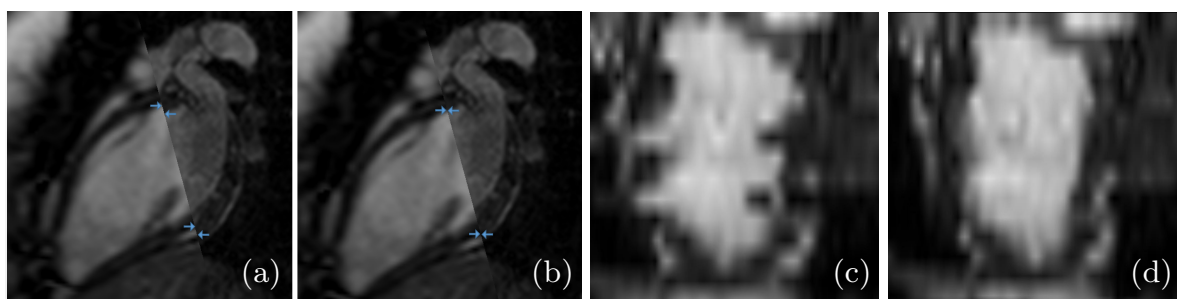

Fig. 3. Example of image registration and alignment: a) misalignment of two different left ventricle images, b) rigid registration of images showing alignment, c) short axis stack of the left ventricle (bright blood pool) where slices are misaligned due to respiratory motion, d) re-aligned short axis stack.

Visualizing the results of these reconstruction operations is a necessary component of ensuring data correctness as automatic registration algorithms do not always produce optimal results. Eidolon allows for easy observation of the results in three dimensions and time, providing a level of user feedback two dimensional and single image viewing tools do not.

\subsection{Motion Estimation}

Motion estimation from time-dependent images is performed using IRTK's builtin routines [111] based on non-rigid registration. This results in deformation fields which are applied to a user-specified geometric mesh giving quantitative motion data.

The motion tracking process is procedurally more complicated than rigid registration and is time-intensive. To manage this process, and automate the deformation of geometry using these files, Eidolon includes the module and UI support for invoking IRTK correctly. Within a single environment, the user can import and prepare motion image data, extract motion, and apply the resulting deformation to geometry without having to translate IRTK specific outputs.

Figure 4 shows the result of this process done with the motion extracted from a 3D Tagged MRI [9] sequence of a healthy left ventricle applied on its mesh. This particular type of image is rich in 3D motion information and used by engineers, discussed further in the results. The $2 \mathrm{D}$ and $3 \mathrm{D}$ viewing windows of Eidolon provides different but necessary views for researchers to interpret the derived motion.

\subsection{Clinical Output}

Deriving clinically-relevant results from the motion geometry is the final stage of the biomedical workflow. Strain, average displacement, wall thickness, volume changes, and other indicators can be calculated from the left ventricle geometry mesh, which are relevant to assessing cardiac function. Additionally, these are the 


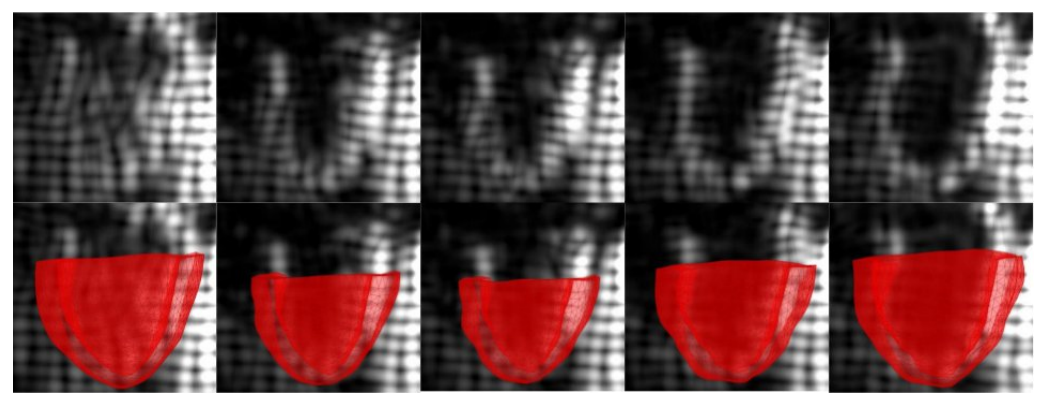

Fig. 4. Deformation of a healthy left ventricle with motion estimation from a 3D Tagged MRI sequence. The top pictures hows the image itself, while the bottom overlays the geometric mesh.

type of image derived metrics that can help stratify patients in disease groups, a common technique for understanding aetiology and potential treatment efficacy.

Eidolon simplifies and streamlines how the tasks leading to these results are carried out by enabling complex operations through simple interfaces, and providing rapid assessment of results through its visualization features. Some of the derived clinical quantities are more sensitive to noise, however the ability to pre-process the data in Eidolon alleviates some of that burden.

\subsection{D Visualization}

Multi-sequence imaging data represents information captured in specified areas of 3D space (at a particular time) from a common spatial origin. A single 2dimensional image includes spatial transformation data placing each captured pixel in a 3D position. Typically such transformations are in a coordinate system relative to the acquiring scanner (for scanner modalities), therefore multiple acquisition sequences can be expected to exist in the same space. This allows visualization of multiple image series concurrently, however certain considerations must be taken to ensure any image renderings compose together correctly, compose coherently with geometry data, and align in time. Figure 5 illustrates the types of image data which must be rendered together to allow different image acquisition series to be compared.

Rendering images as individual planes in space is a simple matter of representing each image as a quadrilateral with a texture. Intersecting geometry renders correctly together if totally opaque, however applied transparency may introduce artefacts if the order of rendering is incorrect. Rendering a 2D image with 3D volume (Figure 5 (c)) must also take the order into account, however the important issue is how the textured quad is composited with the volume.

Our solution is to employ a volume-slicing technique 2,4 for representing volume objects. Image series are converted into 3D textures and associated with a rendering object which generates geometry planes bisecting the image volume. These planes are parallel with the view direction and slice through the volume at regular intervals. Texture coordinates are assigned to these planes so that 


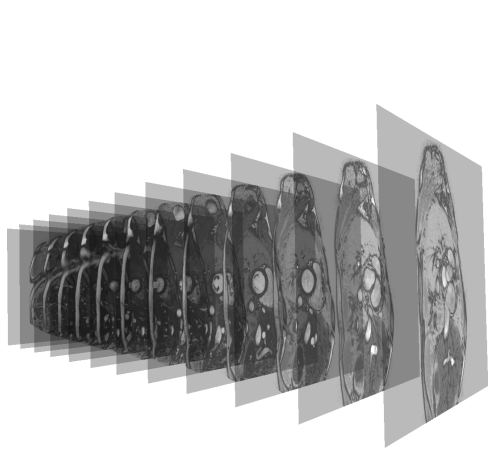

(a)

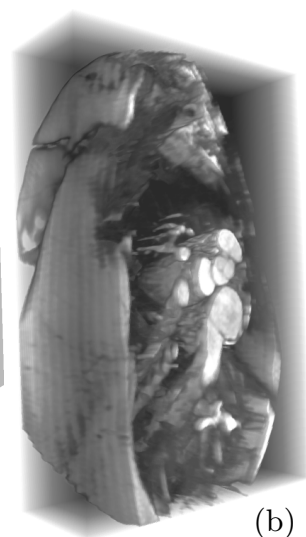

(b)

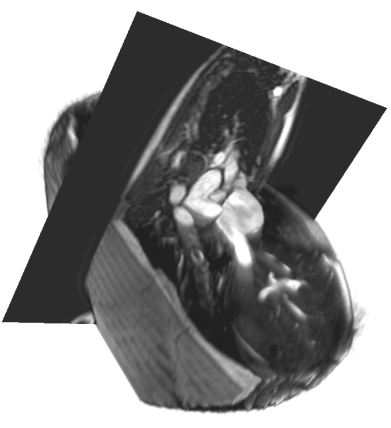

(c)

Fig. 5. Example time-dependent MR images: a) 3D image stack represented as 2D planes uniformly spaced apart and aligned with the LV axis (short axis), b) the same stack data represented as a $3 \mathrm{D}$ volume, c) 2D plane intersecting $3 \mathrm{D}$ volume.
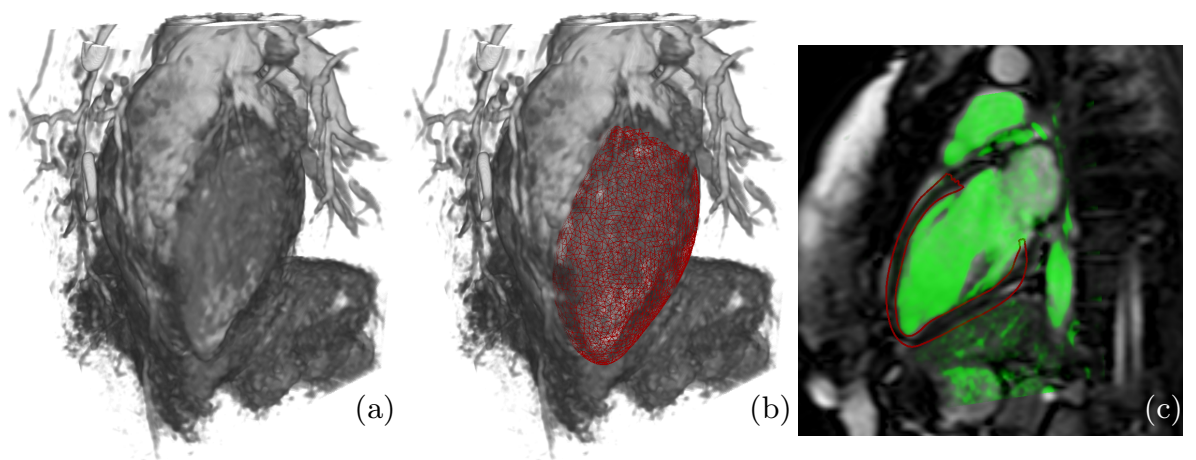

Fig. 6. Image volume of left ventricle, a) MR image only, b) LV segmentation mesh, c) 2D image (greyscale) with sliced volume (green) and sliced mesh (red).

they represent the equivalent slice through the texture space. By rendering this geometry back-to-front a volume is built up which will composite with other geometry in the standard way defined by the rendering pipeline.

A volume image can be combined with 2D images (Figure 5 (c)) and with geometry meshes (Figure 6). The latter figure illustrates a left ventricle mesh derived from an automated segmentation [3] of the image it overlays. The ability to render both multiple images and geometries together is important for assessing the alignment of images which represent the same structures, verifying the segmentation meshes were generated in the correct space, or otherwise visually assessing the correctness of data. 


\subsection{D Visualization}

Identifying structures at specific locations in $3 \mathrm{D}$ is not always practical if the location of interest is surrounded by other detail, so a $2 \mathrm{D}$ view is also important. Further, the 2D view provides a familiar data alignment for medical professionals. The slicing approach has the additional advantage in that a single slice on a desired view plane within a $3 \mathrm{D}$ volume can be taken from the volume and then rendered in a $2 \mathrm{D}$ view. Computationally this is a cheap calculation, requiring only a small geometric object to be created, and when rendering shaders need only calculate values on this geometry.

Figure 6 (c) gives an example of a 2D view showing sliced image and geometry rendered together. A plane is chosen corresponding to the location in space of the greyscale $2 \mathrm{D}$ background image and is then used to calculate a $2 \mathrm{D}$ plane through the volume image, shown in green. The intersection isoline of a left ventricle segmentation geometry with the plane is shown in red, validating that the geometry is a correct segmentation at this location.

\section{Results}

Myocardial strain is a quantity that indicates the effectiveness of ventricular contractions and thus cardiac health. In patients with heart failure, quantifying how synchronously the ventricle contracts is a key clinical metric for therapy response. Further, investigating this quantity compared to the motion seen in healthy subjects can shed light on overall disease mechanisms. Thus, researchers utilizing Eidolon are interested in strain metrics derived from MR images with an ultimate goal of assessing if these metrics can be used for patient stratification.

Historically, strain has been assessed with echocardiography (by speckletracking techniques), however cardiac magnetic resonance (CMR) imaging has increasingly become a gold standard for ventricular volume and function assessment [5]. With CMR, 3D Tagged MRI is the preferred sequence for strain measurements, as it provides rich three dimensional deformation data [9]. Cine MRI is a commonly used sequence in the clinical scenario due to its relatively high spatio-temporal resolution [13. Unfortunately the two dimensional nature of the image modality, and fundamental difficulty in tracking actual material points, limits its capability to capture complex myocardial motion. Eidolon is used to comparatively render different meshes to assess the difference in calculating strain from Cine MRI versus 3D Tagged MRI.

The synergy of multi-modal images and computational geometry provided by Eidolon is shown in Figure 7. By viewing these datasets together with different renderings in space and time, researchers can quickly assess the resulting mesh's quality and ensure the derived strain values accurately characterize the subject.

Figure 8 demonstrates a typical global strain pattern for healthy individuals in the cardiac cycle starting in diastole from 3D Tagged MRI and Cine MRI data preprocessed by Eidolon. The global radial strain has a positive peak since the myocardium is thickening during the cardiac cycle. Conversely, both 


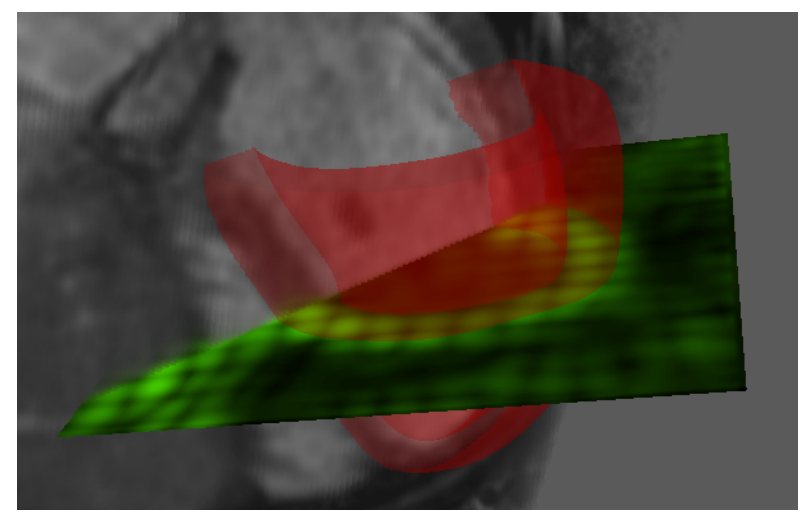

Fig. 7. Visualization of 3D Tagged MRI (green), Cine MRI (black and white) and geometric meshes (red) used for strain analysis in Eidolon. This highlights the image registration and correction capabilities of Eidolon as multi-modal images and computational meshes are made spatially consistent.

the circumferential and longitudinal strains exhibit negative peaks, capturing the cavity volume reduction and axial shortening respectively. The difference in strain calculated from the two modalities clearly shows how Cine MRI lacks longitudinal shortening information as it inaccurately gives a non-physiological strain.

Comparing the strain pattern between a healthy volunteer and dyssynchronous heart failure patient (Figure 9) highlights the significant difference in the peak global strain measures (over 50\%), revealing the inefficient contraction pattern of the patient. The reason behind this decrease is better portrayed in Figure 10 where the global circumferential strain is broken into its mid-ventricle regional components for both the healthy subject and patient. In the healthy subject, the circumferential strain is consistently negative during systole and the peak strain of each region occurs at approximately the same time, which demonstrates synchronicity of contraction. Conversely, the patient's ventricle does not have the same synchronous contraction pattern as evidenced by the spread in circumferential strain across positive and negative values. Thus, the global sum of regional strain is negative when adding strictly negative regional values, whereas the mismatched positive and negative regional patient strains sum closer to zero. The ability to look reliably in more depth at the data, from global to regional quantities, is made possible by the resulting data quality from the Eidolon's preprocessing capabilities.

\section{Discussion}

We have described our visualization software Eidolon and demonstrated its use within the context of a research workflow. The visualization component has been specifically targeted at biomedical applications with a volume rendering 

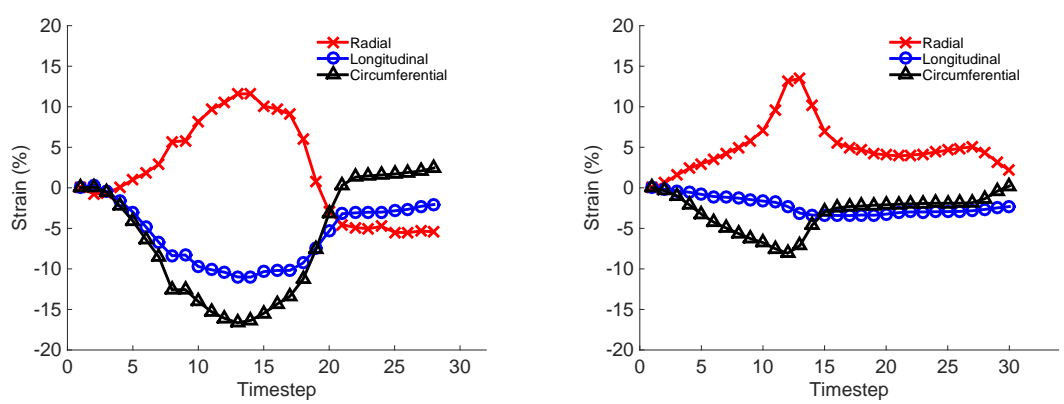

Fig. 8. Comparison of global strain measurements derived from a) 3D tagged MRI and b) Cine MRI, showing a quantitative correspondence between global radial strain and a qualitative correspondence in circumferential strain, both in terms of the peak strain and peak timing. However, it is clear that the two dimensional nature of Cine MRI fails to capture the shortening and thus the resulting longitudinal strain.
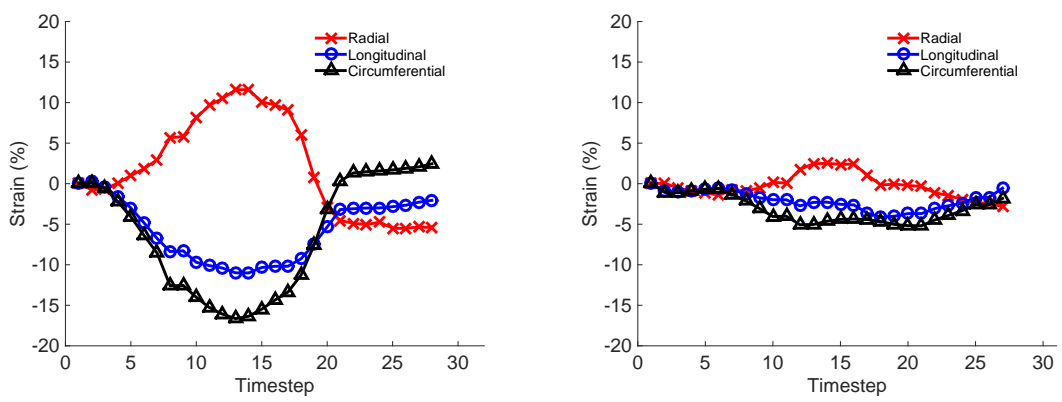

Fig. 9. Comparison of the global strain patterns between a healthy subject (left) and patient with dyssynchronous heart failure (right), showing a significant difference in magnitude for each strain component. This data was calculated from 3D tagged MR images.

approach which emphasizes suitability for 3D as well as 2D rendering of timeresolved image sequences, and a simple accessible underlying implementation.

This approach contrasts with GPU-based raycasting techniques [6]10] which have been the subject of much current research. Although raycasting has certain advantages both aesthetically and in some cases in terms of performance, compositing with arbitrary geometry is not a simple adaptation to the technique. Our slicing implementation uses $\mathrm{Cg}$ [7] shader programs to implement a color transfer function and other features including slicing. Combining these shaders, which are applied to geometry as well as image objects, with a raycasting volume object would also involve a complex implementation.

By using Python, Eidolon can access the wide range of modules which come with the language's runtime, from general purpose application support to user interface creation. Eidolon integrates a command console which allows user access to the internal code and data structures of the visualization module, as well as 

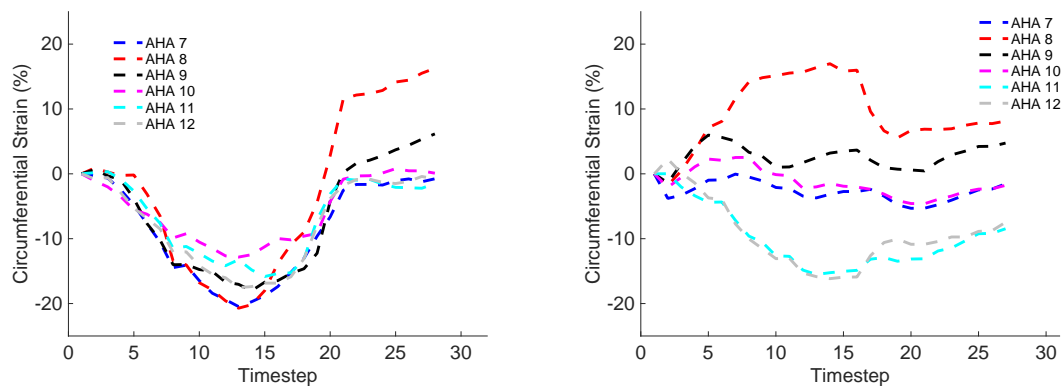

Fig. 10. Dissection of strain values into regional components for a healthy volunteer (left) and patient with dyssynchronous heart failure (right), for AHA regions 7-12.

data processing algorithms. The SciPy libraries, which include image processing and data analysis, are accessible through this console, allowing users to interact with image and geometry data loaded in Eidolon, define new algorithms, generate data, and immediately visualize results within a single interface.

This concept of an all-inclusive environment, made interactive through a programming language, is similar in notion to the IPython(http://www.ipython. org) or the MATLAB environment. Although separate tools exist to perform these functions, our experience has been that the cyclic workflow of data processing, visualization, adjusting parameters, and then repeating, is best enabled by an integrated tool specifically targeted at biomedical research.

In terms of limitations, Eidolon must rely on a multi-language implementation since critical algorithms must be implemented in $\mathrm{C}++$ for speed. Due to the renderer not being compatible with other visualization libraries, functionality for implementing visualizations or interfaces cannot be directly used but must be to some degree re-implemented in Python.

\section{Conclusion and Future Work}

We have presented Eidolon and discussed the critical role an integrated visualization and computational environment plays in biomedical engineering research. Visualizing multiple images with geometry data is shown here to be an important aspect in this research, as a tool for analysis and validation, and the specific properties of our approach best suits research workflows.

Currently, Eidolon is being used in a large research project, VP2HF (http: //vp2hf.eu), which aims to better understand why only $66 \%$ of heart failure patients respond to CRT treatment. Additionally, a current prospective clinical trial aims to perform predictive patient-specific modeling within a decision timeframe of two weeks. Without the integration and computation facilities of Eidolon, adhering to this timeline would be difficult. In other ways we intend to extend the framework Eidolon represents to encompass a greater scope of visualization and computational tasks inherent in these efforts, including visualization of velocity fields and image reconstruction from acquired raw data. 
Acknowledgements This research was partly supported by the National Institute for Health Research (NIHR) Biomedical Research Centre (BRC), and by the NIHR Healthcare Technology Co-operative for Cardiovascular Disease, both at Guy's and St Thomas' NHS Foundation Trust. The research leading to these results has received funding from the EU FP7 for research, technological development and demonstration under grant agreement VP2HF (no 611823), and from BHF New Horizons grant NH/11/5/29058. Views expressed are those of the authors and not necessarily of the NHS, the BHF, the NIHR, or the Dept. of Health.

\section{References}

1. Chandrashekara, R., Mohiaddin, R.H., Rueckert, D.: Analysis of 3D myocardial motion in tagged MR images using nonrigid image registration. IEEE Transactions on Medical Imaging 23(10), 1245-1250 (Oct 2004)

2. Cullip, T.J., Neumann, U.: Accelerating volume reconstruction with 3D texture hardware. Tech. rep., Chapel Hill, NC, USA (1994)

3. Groth, A., Weese, J., Lehmann, H.: Robust left ventricular myocardium segmentation for multi-protocol MR. In: Medical Imaging 2012: Image Processing. vol. 8314, p. 83142S (Feb 2012)

4. Hibbard, W., Santek, D.: Interactivity is the key. In: Proceedings of the 1989 Chapel Hill Workshop on Volume Visualization. pp. 39-43. VVS '89, ACM, New York, NY, USA (1989)

5. Keenan, N.G., Pennell, D.J.: CMR of ventricular function. Echocardiography 24(2), 185-193 (2007)

6. Kruger, J., Westermann, R.: Acceleration techniques for GPU-based volume rendering. In: Proceedings of the 14th IEEE Visualization 2003 (VIS'03). pp. 38-. VIS '03, IEEE Computer Society, Washington, DC, USA (2003)

7. Mark, W.R., Glanville, R.S., Akeley, K., Kilgard, M.J.: Cg: A system for programming graphics hardware in a C-like language. ACM Trans. Graph. 22(3), 896-907 (Jul 2003)

8. Rueckert, D., Sonoda, L.I., Hayes, C., Hill, D.L., Leach, M.O., Hawkes, D.J.: Nonrigid registration using free-form deformations: application to breast MR images. Medical Imaging, IEEE Transactions on 18(8), 712-721 (1999)

9. Rutz, A., Ryf, S., Plein, S., Boesiger, P., Kozerke, S.: Accelerated whole-heart 3D CSPAMM for myocardial motion quantification. Magn Reson Med 59, 755-763 (2008)

10. Scharsach, H.: Advanced GPU raycasting. In: In Proceedings of CESCG 2005. pp. 69-76 (2005)

11. Shi, W., Zhuang, X., Wang, H., Duckett, S., Luong, D., Tobon-Gomez, C., Tung, K., Edwards, P., Rhode, K., Razavi, R., Ourselin, S., Rueckert, D.: A comprehensive cardiac motion estimation framework using both untagged and 3D tagged MR images based on non-rigid registration. IEEE Trans. Med. Imaging 31(6), 1263$1275(2012)$

12. Studholme, C., Hill, D., Hawkes, D.: An overlap invariant entropy measure of 3D medical image alignment. Pattern Recognition 32(1), 71 - 86 (1999)

13. Walsh, T.F., Hundley, W.G.: Assessment of ventricular function with cardiovascular magnetic resonance. Magnetic resonance imaging clinics of North America 15(4), 487-504 (2007) 